\begin{tabular}{|c|c|}
\hline Citation & $\begin{array}{l}\text { Casaer MP, Van den Berghe G, }(2020) \\
\text { Indirect calorimetry: A faithful guide for nutrition therapy, or a fascinating } \\
\text { research tool? } \\
\text { Clinical Nutrition, } 2020 \text { Dec 11, S0261-5614 (20)30666-X (ahead of print) }\end{array}$ \\
\hline Archived version & $\begin{array}{l}\text { Author manuscript: the content is identical to the content of the published } \\
\text { paper, but without the final typesetting by the publisher }\end{array}$ \\
\hline Published version & http://dx.doi.org/10.1016/j.clnu.2020.12.007 \\
\hline Journal homepage & $\underline{\text { Clinical Nutrition }}$ \\
\hline Author contact & $\begin{array}{l}\text { greet.vandenberghe@kuleuven.be } \\
+32(0) 16344021\end{array}$ \\
\hline IR & https://lirias2. kuleuven.be/viewobject.html?cid=1\&id=3365303 \\
\hline
\end{tabular}

(article begins on next page) 


\title{
Indirect Calorimetry: a faithful guide for nutrition therapy, or a fascinating research tool?
}

\author{
Michael P. Casaer*, Greet Van den Berghe and Jan Gunst
}

From the Laboratory of Intensive Care Medicine, Department of Cellular and Molecular Medicine, KU Leuven, Belgium

*Correspondence to Michael.casaer@uzleuven.be

Dear editor,

Singer et al. recently published the results of the long-awaited TICACOS-International randomized controlled trial (RCT), which found no benefit of indirect calorimetry (IC)-guided feeding in mixed intensive care unit (ICU) patients, as compared to feeding up to a calculated energy target. ${ }^{1}$ The authors conclude that IC-guided feeding may still be superior, because (1) the RCT was stopped prematurely and (2) aggregation with smaller, neutral IC-RCTs might reveal a beneficial trend.

Yet, this giant multicenter effort may question the feasibility of IC-guided feeding. Indeed, ICexperts in 7 centers failed to include more than 417 patients over 6 years, with only 339 patients included in per-protocol analysis. Could the authors report the total number of patients admitted to the 7 ICUs over 6 years, and comment why only a (presumably small) fraction of these have been screened. Could the authors also provide per exclusion criterion, the respective number of patients excluded? Additionally, the number of missing IC-data in included patients (and reasons for missing) would be very informative. Moreover, it is not clear if per-protocol groups were comparable. Could the authors provide the reasons for post-randomization exclusion per study-arm (figure 1), the reasons of ICU-admission per group, and the number of included men/women, which does not correspond with the total number of patients?

Objective evaluation of the presumed beneficial trend in this neutral RCT requires rigorous data. Therefore, in the Kaplan-Meier curve, could the authors provide the numbers at risk and time-unit on the X-axis, and label the study-groups? Strikingly, the 6.00 to 12.00 time-window appears free of events and of censoring. How is this explained clinically? In table 3, 3-month mortality is lower than ICU-mortality (with average ICU-stay of 12-15 days) and higher than 6-month mortality. Moreover, mortalities in the abstract do not seem to agree with these numbers. If aggregated data from the 4 IC-RCT's would suggest improved survival, it is also important to understand the underlying causes. Could the authors provide statistical evaluation of the evolution in SOFA-scores, which appear higher in the IC-group (appendix 4.16)? Could the authors also clarify the number of infections (different number in text versus table)?

A potential confounding factor is the quality of blood glucose control. ${ }^{2}$ Indeed, the highest glucose concentrations were higher in the IC-group. Poorer glucose control may have counteracted any benefit of IC-guided feeding. Could the authors also report the mean glucose concentrations over time and the time-in-blood-glucose-target range, as well as the total amount of glucose/fat administration? Finally, could the authors clarify the timing of initiation of parenteral nutrition?

Undoubtedly, this additional information will allow to judge whether IC remains a fascinating research tool or constitutes an essential guide in clinical practice. 


\section{References}

1. Singer P, De Waele E, Sanchez C, et al. TICACOS international: A multi-center, randomized, prospective controlled study comparing tight calorie control versus Liberal calorie administration study. Clin Nutr 2020.

2. Gunst J, De Bruyn A, Van den Berghe G. Glucose control in the ICU. Curr Opin Anaesthesiol 2019;32:156-62.

\section{Conflicts of interest}

The authors have no conflict of interest to declare regarding this manuscript.

\section{Funding}

MPC holds a postdoctoral research fellowship supported by the Research Foundation - Flanders $(1832817 \mathrm{~N})$. JG holds a postdoctoral research fellowship supported by the Clinical Research and Education Council of the University Hospitals Leuven. MPC and JG receive research funding from from $\mathrm{KU}$ Leuven $(\mathrm{C} 24 / 17 / 070) \mathrm{GVdB}$ receives structural research financing via the Methusalem program funded by the Flemish government through the university of Leuven (METH14/06) and a European Research Council Advanced Grant (ERC-2017-ADG-785809).

\section{Author contributions}

MPC wrote the first draft of the manuscript which was revised and approved by all authors. 